\title{
Posizionamento del vino Italiano nel mercato Norvegese
}

\author{
Alessio Marco Lazzari, Andrea Dal Vecchio, Danielle Borra, e Stefano Massaglia \\ DISAFA, Università di Torino, Largo Braccini, 2, 10095 Grugliasco (TO), Italy
}

\begin{abstract}
Sintesi. Il mercato norvegese si sta configurando sempre più strategico per il comparto vinicolo italiano: le esportazioni di vino nel quinquennio 2010-2014 sono aumentate in valore dell' $80 \%$ e la loro incidenza sul totale delle esportazioni italiane verso la Norvegia è passato dal 4,3\% al 6\%. L'Italia è il paese che nel mercato del vino Norvegese è cresciuto maggiormente nel quinquennio considerato. In tutte le categorie (rossi, bianchi, rosati e spumanti) i vini italiani fanno registrare incrementi in termini di volume e fatturato generato. La ricerca è stata realizzata analizzando i dati di vendita (volumi e fatturato) del Monopolio norvegese e che ha l'esclusiva di vendita al dettaglio di vini ed alcoolici. Nello studio viene inoltre analizzata l'incidenza dei formati bag-in Box che con un rapido sviluppo sono arrivati ad incidere per oltre la metà nel volume venduto e si stanno sviluppando sempre più nella fascia di prezzo media.
\end{abstract}

\section{I consumi di vino in Norvegia}

Il consumo di vino è in costante aumento in Norvegia attestandosi nel 2014 a 14 litri procapite e erodendo quote di mercato verso altre bevande come la birra più legate agli stili di consumo tradizionali (quest'ultima bevanda rappresenta circa $80 \%$ di tutti gli alcolici venduti in Norvegia).

Il mercato norvegese si sta configurando sempre più strategico per il comparto vinicolo Italiano: le esportazioni di vino nel quinquennio 2010-2014 sono aumentate in valore dell' $80 \%$ e la loro incidenza sul totale delle esportazioni italiane verso la Norvegia è passato dal 4,3\% al $6 \%$.

Il mercato vinicolo norvegese è governato dal Ministero della Salute e degli Affari Sociali e dal Monopolio statale [1,2], che gestisce la vendita al dettaglio ed è la sola agenzia autorizzata a vendere vino e altre bevande alcoliche con gradazione superiore al $4 \%$ al consumatore tramite una rete di 297 punti vendita distribuiti sul territorio nazionale.

Nel sistema del monopolio norvegese, Vinmonopolet può acquistare il vino solo attraverso importatori e/o grossisti e non direttamente dai produttori.

La scelta del vino in questa struttura di mercato dipende fortemente dal prezzo, dalla qualità del prodotto e dalla percezione che i consumatori hanno della denominazione o del paese d'origine.

Negli ultimi cinque anni questo mercato è stato uno dei più dinamici e interessanti a livello mondiale.

Nel 2010 le importazioni complessive di vino raggiungevano un valore di 2.115 milioni di corone norvegesi mentre nel 2014 sono arrivate ad un valore di 2.897 milioni di corone, con un incremento del $37 \%$.

L'incremento è stato ancora maggiore per i vini Italiani: da 638 a 1.044 milioni di corone, permettendo così alle produzioni enologiche italiane di aumentare la quota di mercato dal 30\% al 36\% e superando così stabilmente la quota detenuta dalle produzioni provenienti dalla Francia, pari rispettivamente al $29 \%$ e $30 \%$ ad inizio e fine del quinquennio.

I dati sulle importazioni complessive permettono di distinguere fra importazioni di vino in bottiglia e vino sfuso: nel primo caso l'incremento complessivo in termini di fatturato è stato del $37 \%$, con un incremento delle bottiglie provenienti dall'Italia che è stato pari al $+50 \%$. Per quanto concerne il vino sfuso (bag-in-box) l'incremento complessivo in fatturato delle importazioni è stato del $24 \%$, con una progressione dei vini provenienti dall'Italia del $+56 \%$. Per quanto riguarda l'incidenza del vino sfuso sul complessivo del volume generato in questo comparto, le quantità vendute di vino in bag-in box negli ultimi anni ha superato il vino in bottiglia.

Il consumatore norvegese è fortemente orientato verso i vini rossi che rappresentano nei quantitativi circa il $65 \%$ delle vendite contro il circa $35 \%$ complessivamente consumato di vini bianchi, rosati e spumanti.

\section{Metodologia}

L'analisi del mercato Norvegese è stata effettuata sul periodo di riferimento 2010-2014, analizzando le banche dati relative alle vendite effettuate presso i punti vendita del Monopolio fornite da Vinmonopolet agli importatori.

L'elaborazione di tali dati ha reso possibile approfondire la ricerca sui volumi venduti e il fatturato generato dalle vendite per tipologia di vino e per tipologia di formato approfondendo l'indagine sulle etichette effettivamente vendute nei punti vendita della catena di stato (più di 11.200 etichette di vino, Italiano ed estero).

I risultati presentati in questo lavoro, illustrano l'evoluzione delle vendite ed i trend più significativi individuati nell' arco temporale considerato. 
Tabella 1. Volumi delle vendite (1) nei punti vendita Vinmonopolet.

\begin{tabular}{|l|l|l|l|}
\hline Nazione & Anno 2010 & Anno 2014 & Var. \% \\
\hline Italia & 16.761 .072 & 21.841 .947 & $30 \%$ \\
\hline Francia & 11.460 .598 & 11.445 .169 & $0 \%$ \\
\hline Spagna & 7.647 .531 & 6.890 .262 & $-10 \%$ \\
\hline Germania & 5.140 .511 & 5.438 .822 & $6 \%$ \\
\hline Cile & 4.445 .672 & 4.909 .942 & $10 \%$ \\
\hline Australia & 6.725 .449 & 4.545 .834 & $-32 \%$ \\
\hline USA & 2.099 .656 & 2.757 .340 & $31 \%$ \\
\hline Portogallo & 2.165 .763 & 2.310 .757 & $7 \%$ \\
\hline Argentina & 2.866 .851 & 1.444 .398 & $-5 \%$ \\
\hline Sud Africa & 1.749 .823 & 1.403 .883 & $-2 \%$ \\
\hline Altri & 1.753 .015 & 1.952 .992 & $11 \%$ \\
\hline Totale & 62.815 .941 & 64.941 .346 & $3 \%$ \\
\hline
\end{tabular}

Tabella 2. Fatturati (Euro) nei punti vendita Vinmonopolet.

\begin{tabular}{|l|l|l|l|}
\hline Nazione & Anno 2010 & Anno 2014 & Var. \% \\
\hline Italia & 295.620 .688 & 421.036 .519 & $42 \%$ \\
\hline Francia & 215.966 .472 & 241.078 .617 & $12 \%$ \\
\hline Spagna & 124.122 .710 & 122.107 .597 & $-2 \%$ \\
\hline Germania & 75.295 .635 & 88.578 .484 & $18 \%$ \\
\hline Australia & 106.122 .805 & 75.091 .943 & $-29 \%$ \\
\hline Cile & 62.782 .392 & 72.297 .903 & $15 \%$ \\
\hline USA & 30.816 .896 & 44.997 .306 & $46 \%$ \\
\hline Portogallo & 32.802 .837 & 37.598 .311 & $15 \%$ \\
\hline Argentina & 43.065 .906 & 24.673 .414 & $-43 \%$ \\
\hline Sud Africa & 27.509 .903 & 22.841 .150 & $-17 \%$ \\
\hline Altri & 28.057 .548 & 35.406 .388 & $26 \%$ \\
\hline Totale & 1.042 .163 .792 & 1.185 .707 .632 & $14 \%$ \\
\hline
\end{tabular}

I valori relativi ai fatturati sono espressi in euro, al tasso di cambio fissato convenzionalmente alla data del $10 / 01 / 2015(1 \mathrm{NOK}=8.19 €)$, e si riferiscono al prezzo di vendita al consumo medio nell'anno di riferimento comprensivo delle tasse e accise.

\section{Vendite di vino complessive presso $i$ punti vendita del Monopolio}

Le vendite di vino nei punti vendita del Monopolio Norvegese nel periodo 2010-14 (Tabb. 1 e 2) sono cresciute del $3 \%$ in volume e del $14 \%$ in fatturato sviluppato. In termini di crescita assoluta l'Italia è il primo paese per volumi e fatturato generato con una crescita nel periodo di indagine del $30 \%$ nei volumi e del $42 \%$ nel fatturato. Nel complesso i principali paesi produttori mantengono i volumi e i fatturati la crescita del mercato è spinta principalmente dall'Italia

\section{Vini rossi}

\subsection{Vendite complessive di vini rossi}

Tra il 2010 e il 2014 (Tabb. 3 e 4) il totale volume di vino rosso venduto in Norvegia diminuisce del 5,3\% in volume e del $6,3 \%$ in fatturato.

Il trend più significativo rispetto al vino rosso è rappresentato da un lato dal calo dei volumi e dei fatturati
Tabella 3. Volumi (1) delle vendite di vino rosso.

\begin{tabular}{|l|l|l|l|}
\hline Nazione & Anno 2010 & Anno 2014 & Var. \% \\
\hline Italia & 14.076 .500 & 17.432 .420 & $23,80 \%$ \\
\hline Francia & 6.173 .200 & 4.759 .977 & $-22,90 \%$ \\
\hline Spagna & 6.049 .200 & 5.241 .433 & $-13,40 \%$ \\
\hline Australia & 5.446 .600 & 3.181 .012 & $-41,60 \%$ \\
\hline Cile & 3.801 .500 & 3.813 .412 & $0,30 \%$ \\
\hline Argentina & 2.225 .000 & 1.009 .141 & $-54,60 \%$ \\
\hline Portogallo & 1.902 .400 & 1.889 .603 & $-0,70 \%$ \\
\hline USA & 1.772 .100 & 2.295 .991 & $29,60 \%$ \\
\hline Sud Africa & 1.466 .800 & 1.060 .304 & $-27,70 \%$ \\
\hline Altri & 273.200 & 232.101 & $-15,00 \%$ \\
\hline Totale & 43.186 .500 & 40.915 .394 & $-5,30 \%$ \\
\hline
\end{tabular}

Tabella 4. Fatturato (Euro) vino rosso.

\begin{tabular}{|l|l|l|l|}
\hline Nazione & Anno 2010 & Anno 2014 & Var. \% \\
\hline Italia & 249.599 .927 & $€ 337.479 .608$ & $35,20 \%$ \\
\hline Francia & 103.686 .761 & $€ 91.025 .041$ & $-12,20 \%$ \\
\hline Spagna & 98.201 .445 & $€ 92.224 .714$ & $-6,10 \%$ \\
\hline Australia & 85.687 .654 & $€ 52.616 .463$ & $-38,60 \%$ \\
\hline Cile & 53.252 .009 & $€ 55.503 .043$ & $4,20 \%$ \\
\hline Argentina & 33.930 .794 & 18.003 .234 & $-46,90 \%$ \\
\hline Portogallo & 28.505 .472 & 30.674 .355 & $7,60 \%$ \\
\hline USA & 26.021 .899 & 37.574 .303 & $44,40 \%$ \\
\hline Sud Africa & 22.993 .829 & 17.380 .791 & $-24,40 \%$ \\
\hline Altri & 4.500 .917 & 5.196 .267 & $15,40 \%$ \\
\hline Totale & 50.826 .000 & 47.402 .534 & $-6,70 \%$ \\
\hline
\end{tabular}

Tabella 5. Volumi (1) vino rosso-regioni Italiane.

\begin{tabular}{|l|l|l|l|}
\hline Regione & Anno 2010 & Anno 2014 & Var. \% \\
\hline Puglia & 4.603 .715 & 4.181 .535 & $-9,20 \%$ \\
\hline Veneto & 2.926 .814 & 6.373 .942 & $117,80 \%$ \\
\hline Piemonte & 2.231 .162 & 2.613 .620 & $17,10 \%$ \\
\hline Abruzzo & 1.828 .304 & 1.502 .502 & $-17,80 \%$ \\
\hline Toscana & 1.735 .176 & 1.902 .593 & $9,60 \%$ \\
\hline Sicilia & 330.402 & 356.398 & $7,90 \%$ \\
\hline Altre regioni & 420.888 & 501.829 & $19,20 \%$ \\
\hline Italia & 14.076 .461 & 17.432 .420 & $23,80 \%$ \\
\hline
\end{tabular}

di tutti i paesi produttori, ad eccezione degli Stati Uniti e dell'Italia; dall'altro che l'Italia è il paese che fa cresce volume e fatturato per il vino rosso in maniera più forte confermandosi al primo posto in termini assoluti sia nel 2010 che nel 2014 e con una crescita del $23,8 \%$ per volume venduto e del $35,2 \%$ nel fatturato.

Notevoli le quote di mercato perse da storici paesi competitor dell'Italia sui mercati esteri per il vino rosso, come la Francia che perde $22,9 \%$ dei volumi e il $12 \%$ del fatturato, la Spagna -13.4 volume e $-6 \%$ di fatturato.

\subsection{Vendite di vini rossi italiani}

Tra le regioni Italiane produttrici di vino rosso nel periodo 2010-14 (Tabb. 5 e 6) l'aumento maggiore in termini di volume e fatturato si registrano dal Veneto con un aumento del volume venduto $117,8 \%$ e del fatturato del $118 \%$. Le 
Tabella 6. Fatturato (Euro) vino rosso-regioni Italiane.

\begin{tabular}{|l|l|l|l|}
\hline Regione & Anno 2010 & Anno 2014 & Var. \% \\
\hline Veneto & 59.922 .458 & 130.688 .554 & $118,10 \%$ \\
\hline Puglia & 71.197 .424 & 69.987 .986 & $-1,70 \%$ \\
\hline Piemonte & 43.710 .254 & 56.027 .903 & $28,20 \%$ \\
\hline Toscana & 32.703 .847 & 38.338 .526 & $17,20 \%$ \\
\hline Abruzzo & 29.145 .688 & 25.970 .507 & $-10,90 \%$ \\
\hline Altre regioni & 7.069 .521 & 9.143 .029 & $29,30 \%$ \\
\hline Sicilia & 5.850 .735 & 7.323 .103 & $25,20 \%$ \\
\hline Italia & 249.599 .927 & 337.479 .608 & $35,20 \%$ \\
\hline
\end{tabular}

Tabella 7. Volumi (1) vino rosso bag-in-box.

\begin{tabular}{|l|l|l|l|}
\hline Nazione & Anno 2010 & Anno 2014 & Var. \% \\
\hline Italia & 7.778 .500 & 10.000 .049 & $28,60 \%$ \\
\hline Spagna & 3.016 .200 & 2.823 .324 & $-6,40 \%$ \\
\hline Francia & 3.788 .300 & 2.665 .175 & $-29,60 \%$ \\
\hline Cile & 2.389 .100 & 2.613 .197 & $9,40 \%$ \\
\hline Australia & 3.805 .400 & 2.218 .932 & $-41,70 \%$ \\
\hline USA & 1.357 .000 & 1.869 .015 & $37,70 \%$ \\
\hline Portogallo & 998.200 & 1.032 .316 & $3,40 \%$ \\
\hline Sud Africa & 1.086 .400 & 862.073 & $-20,60 \%$ \\
\hline Argentina & 1.157 .400 & 341.277 & $-70,50 \%$ \\
\hline Altri & 19.600 & 0 & nd \\
\hline Totale & 25.396 .100 & 24.425 .358 & $-3,80 \%$ \\
\hline
\end{tabular}

altre regioni ad eccezione dell'Abruzzo fanno registrare anch'esse trend in aumento in termini di volumi e fatturato.

\subsubsection{Vini rossi italiani in bag-in-box}

L'incidenza del formato bag-in-box (Tabb. 7 e 8) per il vino rosso nel periodo 2010-14 è molto importante rappresentando circa il $50 \%$ del volume venduto per questa categoria di vino in Norvegia.

Nel formato bag-in-box per il vino rosso, il trend è simile a quello generale per la stessa categoria di vino riferito ai paesi produttori, con una diminuzione generale dei volumi venduti e dei fatturati, ovvero anche al formato bag-in-box si cerca la qualità del prodotto, e i consumatori sono disposti a spendere di più.

L'Italia nel formato bag-in-box rappresenta la prima nazione per volumi venduti $+28,6 \%$ e fatturati generati con un $+39,9 \%$, quindi anche per il vino rosso Italiano venduto in questo formato il trend è un aumentato nel volume venduto percepito come di maggiore qualità.

\section{Vini bianchi}

\subsection{Vendite complessive di vini bianchi}

I principali paesi per la categoria vino bianco per fatturato e volume sono Germania, Francia e Italia. Per questa categoria nel periodo 2010-14 (Tabb. 9 e 10) il trend generale è in crescita volume $+13,5 \%$ e fatturato $+25,2 \%$. $\mathrm{Ci}$ sono inoltre trend interessanti riguardo paesi emergenti che fanno segnare tassi di crescita molto alti come Nuova Zelanda, Cile e Sud Africa.
Tabella 8. Fatturato (Euro) vino rosso bag-in-box.

\begin{tabular}{|l|l|l|l|}
\hline Nazione & Anno 2010 & Anno 2014 & Var. \% \\
\hline Italia & 116.179 .970 & 162.500 .013 & $39,90 \%$ \\
\hline Spagna & 40.672 .738 & 41.935 .244 & $3,10 \%$ \\
\hline Francia & 52.946 .384 & 40.852 .496 & $-22,80 \%$ \\
\hline Cile & 31.086 .928 & 35.589 .363 & $14,50 \%$ \\
\hline Australia & 55.772 .505 & 33.919 .424 & $-39,20 \%$ \\
\hline USA & 18.426 .295 & 28.727 .130 & $55,90 \%$ \\
\hline Portogallo & 13.646 .323 & 15.260 .160 & $11,80 \%$ \\
\hline Sud Africa & 15.653 .784 & 13.016 .791 & $-16,80 \%$ \\
\hline Argentina & 16.053 .395 & 5.081 .236 & $-68,30 \%$ \\
\hline Altri & 252.000 & 0 & nd \\
\hline Totale & 360.690 .322 & 376.881 .857 & $4,50 \%$ \\
\hline
\end{tabular}

Tabella 9. Volumi (1) vino bianco.

\begin{tabular}{|l|l|l|l|}
\hline Nazione & Anno 2010 & Anno 2014 & Var. \% \\
\hline Germania & 5.055 .276 & 5.361 .386 & $6,06 \%$ \\
\hline Francia & 4.320 .688 & 5.056 .557 & $17,03 \%$ \\
\hline Italia & 1.605 .590 & 2.087 .089 & $29,99 \%$ \\
\hline Australia & 1.103 .356 & 1.129 .266 & $2,35 \%$ \\
\hline Cile & 571.695 & 939.953 & $64,42 \%$ \\
\hline Ungheria & 887.908 & 728.474 & $-17,96 \%$ \\
\hline Nuova Zelanda & 108.757 & 495.819 & $355,90 \%$ \\
\hline Spagna & 492.549 & 448.557 & $-8,93 \%$ \\
\hline Argentina & 621.923 & 432.507 & $-30,46 \%$ \\
\hline Austria & 363.289 & 362.166 & $-0,31 \%$ \\
\hline Portogallo & 149.739 & 359.665 & $140,19 \%$ \\
\hline Sud Africa & 279.248 & 337.851 & $20,99 \%$ \\
\hline USA & 173.511 & 121.761 & $-29,83 \%$ \\
\hline Altri & 19.524 & 13.121 & $-32,80 \%$ \\
\hline Totale & 15.753 .053 & 17.874 .172 & $13,46 \%$ \\
\hline
\end{tabular}

Tabella 10. Fatturato (Euro) vino bianco.

\begin{tabular}{|l|l|l|l|}
\hline Nazione & Anno 2010 & Anno 2014 & Var. \% \\
\hline Francia & 81.068 .196 & 102.480 .807 & $26,41 \%$ \\
\hline Germania & 73.731 .379 & 56.856 .666 & $-22,89 \%$ \\
\hline Italia & 26.450 .365 & 36.886 .028 & $39,45 \%$ \\
\hline Australia & 17.307 .187 & 18.429 .963 & $6,49 \%$ \\
\hline Cile & 8.395 .217 & 14.285 .507 & $70,16 \%$ \\
\hline Ungheria & 12.581 .901 & 10.807 .667 & $-14,10 \%$ \\
\hline Nuova Zelanda & 2.338 .064 & 9.810 .353 & $319,59 \%$ \\
\hline Spagna & 8.027 .290 & 8.012 .204 & $-0,19 \%$ \\
\hline Austria & 6.486 .775 & 7.014 .702 & $8,14 \%$ \\
\hline Argentina & 8.891 .438 & 6.630 .352 & $-25,43 \%$ \\
\hline Portogallo & 2.456 .442 & 5.830 .816 & $137,37 \%$ \\
\hline Sud Africa & 4.433 .224 & 5.334 .173 & $20,32 \%$ \\
\hline USA & 2.646 .609 & 2.160 .414 & $-18,37 \%$ \\
\hline Altri & 309.040 & 326.776 & $5,74 \%$ \\
\hline Totale & 7.327 .003 & 284.866 .428 & $25,25 \%$ \\
\hline
\end{tabular}

L'Italia cresce $+30 \%$ nei volumi venduti e del 39, $\%$ nel fatturato in questa categoria confermando il suo terzo posto nei paesi produttori di vino bianco venduto in Norvegia.

\subsection{Vendite di vini bianchi italiani}

La principale regione Italiana per il vino bianco in valore assoluto per volume e fatturato è il Veneto, con circa 
Tabella 11. Volumi (1) vino bianco italiano.

\begin{tabular}{|l|l|l|l|}
\hline Regione & Anno 2010 & Anno 2014 & Var. \% \\
\hline Veneto & 820.016 & 1.273 .004 & $55,24 \%$ \\
\hline Abruzzo & 47.367 & 179.597 & $279,16 \%$ \\
\hline Trentino Alto Adige & 116.058 & 177.004 & $52,51 \%$ \\
\hline Puglia & 161.320 & 151.786 & $-5,91 \%$ \\
\hline Sicilia & 70.646 & 74.895 & $6,01 \%$ \\
\hline Piemonte & 59.461 & 67.132 & $12,90 \%$ \\
\hline Friuli Venezia Giulia & 126.169 & 61.435 & $-51,31 \%$ \\
\hline Altre regioni & 251.920 & 102.236 & $-59,42 \%$ \\
\hline Totale & 832.941 & 2.087 .089 & $150,57 \%$ \\
\hline
\end{tabular}

Tabella 12. Fatturato (Euro) vino bianco italiano.

\begin{tabular}{|l|l|l|l|}
\hline Regione & Anno 2010 & Anno 2014 & Var. \% \\
\hline Veneto & 13.559 .915 & 22.141 .174 & $63,28 \%$ \\
\hline Abruzzo Alto & 700.827 & 3.066 .481 & $337,55 \%$ \\
\hline $\begin{array}{l}\text { Trentino } \\
\text { Adige }\end{array}$ & 1.931 .180 & 2.964 .089 & $53,49 \%$ \\
\hline Puglia & 2.506 .606 & 2.584 .267 & $3,10 \%$ \\
\hline Piemonte & 1.109 .964 & 1.365 .017 & $22,98 \%$ \\
\hline Sicilia & 1.201 .448 & 1.330 .237 & $10,72 \%$ \\
\hline Friuli V. G. & 2.025 .142 & 1.182 .588 & $-41,60 \%$ \\
\hline Altre regioni & 4.116 .110 & 2.252 .174 & $-45,28 \%$ \\
\hline Totale & 27.151 .192 & 36.886 .027 & $35,85 \%$ \\
\hline
\end{tabular}

Tabella 13. Volumi (1) vino bianco bag-in-box.

\begin{tabular}{|l|l|l|l|}
\hline Nazione & Anno 2010 & Anno 2014 & Var. \% \\
\hline Francia & 2.272 .093 & 2.670 .645 & $17,54 \%$ \\
\hline Germania & 2.641 .235 & 2.661 .880 & $0,78 \%$ \\
\hline Italia & 639.798 & 1.038 .298 & $62,29 \%$ \\
\hline Australia & 741.772 & 812.508 & $9,54 \%$ \\
\hline Ungheria & 762.786 & 692.883 & $-9,16 \%$ \\
\hline Argentina & 406.604 & 301.853 & $-25,76 \%$ \\
\hline Sud Africa & 208.352 & 277.316 & $33,10 \%$ \\
\hline Austria & 246.462 & 208.713 & $-15,32 \%$ \\
\hline Spagna & 76.526 & 86.257 & $12,72 \%$ \\
\hline Cile & 300.245 & 49.605 & $-83,48 \%$ \\
\hline Altri & 98.224 & 165.553 & $68,55 \%$ \\
\hline Totale & 8.394 .097 & 8.965 .511 & $6,81 \%$ \\
\hline
\end{tabular}

la metà del volume di vino bianco italiano venduto in Norvegia e nel periodo di riferimento (tabb. 11 e 12) cresce ancora del $55,2 \%$ in volume e $63,3 \%$ in fatturato. Ottime performance vengono anche da Abruzzo e Trentino, mentre il calo più forte si registra per il Friuli.

\subsubsection{Vini bianchi italiani in bag-in-box}

Il formato bag-in-box per la categoria vino bianco vale in Norvegia circa un terzo del volume venduto. Nel periodo di riferimento (Tabb. 13 e 14) il trend è +6.8 nei volumi e $+20,4 \%$ nei fatturati generati da questo formato, a indicare che anche per questa categoria di vino come per il rosso, nel mercato norvegese la tendenza è quella di dare un valore maggiore alla quantità nei formati bag-in-box.

L'Italia è il paese che maggiormente cresce per volumi venduti $+62,3 \%$ e fatturato generato $79,4 \%$, passando
Tabella 14. Fatturato (Euro) vino bianco bag-in-box.

\begin{tabular}{|l|l|l|l|}
\hline Nazione & Anno 2010 & Anno 2014 & Var. \% \\
\hline Francia & 34.873 .490 & 44.735 .349 & $28,28 \%$ \\
\hline Germania & 33.684 .282 & 37.182 .692 & $10,39 \%$ \\
\hline Italia & 8.986 .423 & 16.127 .959 & $79,47 \%$ \\
\hline Australia & 10.745 .757 & 12.420 .586 & $15,59 \%$ \\
\hline Ungheria & 10.486 .406 & 10.023 .242 & $-4,42 \%$ \\
\hline Cile & 3.864 .111 & 6.720 .695 & $73,93 \%$ \\
\hline Argentina & 5.545 .189 & 4.404 .882 & $-20,56 \%$ \\
\hline Sud Africa & 2.956 .401 & 4.048 .239 & $36,93 \%$ \\
\hline Austria & 3.711 .276 & 3.380 .015 & $-8,93 \%$ \\
\hline Spagna & 1.035 .741 & 1.301 .893 & $25,70 \%$ \\
\hline Altri & 1.529 .657 & 2.526 .170 & $65,15 \%$ \\
\hline Totale & 116.382 .992 & 139.043 .659 & $19,47 \%$ \\
\hline
\end{tabular}

Tabella 15. Volumi (1) vino rosato.

\begin{tabular}{|l|l|l|l|}
\hline Nazione & Anno 2010 & Anno 2014 & Var. \% \\
\hline Francia & 301.077 & 775.030 & $157,42 \%$ \\
\hline Italia & 350.275 & 347.726 & $-0,73 \%$ \\
\hline Austria & 60.375 & 338.105 & $460,01 \%$ \\
\hline Spagna & 214.634 & 213.163 & $-0,69 \%$ \\
\hline Portogallo & 111.092 & 156.065 & $40,48 \%$ \\
\hline USA & 153.431 & 132.071 & $-13,92 \%$ \\
\hline Cile & 71.627 & 59.974 & $-16,27 \%$ \\
\hline Altri & 46.051 & 52.935 & $14,95 \%$ \\
\hline Totale & 1.308 .562 & 2.075 .069 & $58,58 \%$ \\
\hline
\end{tabular}

Tabella 16. Fatturato (Euro) vino rosato.

\begin{tabular}{|l|l|l|l|}
\hline Nazione & Anno 2010 & Anno 2014 & Var. \% \\
\hline Francia & 5.448 .375 & 15.073 .217 & $176,66 \%$ \\
\hline Italia & 6.356 .281 & 6.613 .566 & $4,05 \%$ \\
\hline Austria & 1.194 .051 & 5.237 .462 & $338,63 \%$ \\
\hline Spagna & 3.373 .436 & 3.377 .677 & $0,13 \%$ \\
\hline Portogallo & 1.792 .475 & 2.498 .936 & $39,41 \%$ \\
\hline USA & 2.125 .129 & 2.233 .465 & $5,10 \%$ \\
\hline Cile & 1.120 .708 & 1.302 .641 & $16,23 \%$ \\
\hline Altri & 674.732 & 840.591 & $24,58 \%$ \\
\hline Totale & 22.085 .187 & 37.177 .555 & $68,34 \%$ \\
\hline
\end{tabular}

dalla quarta posizione del 2010 alla terza nel 2014 in valore assoluto dietro a Germani e Francia e davanti all'Ungheria.

\section{Vini rosati}

\subsection{Vendite complessive di vini rosati}

Il mercato del vino rosato in Norvegia nel periodo di riferimento (Tabb. 15 e 16) cresce sia in termini di volumi venduti $+58,5 \%$ che di fatturato $+68,3$. La crescita avviene in tutti i paesi tranne il Cile, USA e Spagna. Il trend di crescita maggiore viene dall'Austria con un aumento del $460 \%$ in volume.

Nella categoria del vino rosato, l'Italia passa da $1^{\circ}$ paese a $2^{\circ}$ dopo la Francia mantenendosi stabile nei volumi e un $+4 \%$. La Francia e l'Austria ( $3^{\circ}$ posto) sono le due nazioni che crescono di più con un aumento nei volumi rispettivamente del $157 \%$ e del $460 \%$. 
Tabella 17. Volumi (1) vino rosatoitaliano.

\begin{tabular}{|l|l|l|l|}
\hline Regione & Anno 2010 & Anno 2014 & Var. \% \\
\hline Piemonte & 98.684 & 133.886 & $\mathbf{3 5 , 6 7 \%}$ \\
\hline Campania & 52.156 & 100.628 & $\mathbf{9 2 , 9 4 \%}$ \\
\hline Abruzzo & 84.311 & 66.700 & $-20,89 \%$ \\
\hline Veneto & 61.204 & 36.252 & $-40,77 \%$ \\
\hline Altre regioni & 53.922 & 70.289 & $\mathbf{3 0 , 3 5 \%}$ \\
\hline Totale & $\mathbf{3 5 0 . 2 7 7}$ & $\mathbf{4 0 7 . 7 5 5}$ & $\mathbf{1 6 , 4 1 \%}$ \\
\hline
\end{tabular}

Tabella 18. Fatturato (Euro) vino rosato italiano.

\begin{tabular}{|l|l|l|l|}
\hline Regione & Anno 2010 & Anno 2014 & Var. \% \\
\hline Piemonte & 1.843 .000 & 2.223 .339 & $20,64 \%$ \\
\hline Campania & 1.190 .877 & 1.980 .030 & $66,27 \%$ \\
\hline Veneto & 1.109 .904 & 890.576 & $-19,76 \%$ \\
\hline Abruzzo & 1.338 .628 & 158.815 & $-88,14 \%$ \\
\hline Altre regioni & 873.871 & $€ 1.360 .806$ & $55,72 \%$ \\
\hline Totale & $€ 6.356 .280$ & 6.613 .566 & $4,05 \%$ \\
\hline
\end{tabular}

Tabella 19. Volumi (1) vino rosato in bag-in-box.

\begin{tabular}{|l|l|l|l|}
\hline Nazione & Anno 2010 & Anno 2014 & Var. \% \\
\hline Francia & 66.048 & 256.922 & $288,99 \%$ \\
\hline USA & 129.871 & 249.141 & $91,84 \%$ \\
\hline Spagna & 60.692 & 109.871 & $81,03 \%$ \\
\hline Italia & 127.257 & 87.135 & $-31,53 \%$ \\
\hline Altri & 89.606 & 141.195 & $57,57 \%$ \\
\hline Totale & 412.782 & 844.264 & $104,53 \%$ \\
\hline
\end{tabular}

Tabella 20. Fatturato (Euro) vino rosato in bag-in-box.

\begin{tabular}{|l|l|l|l|}
\hline Nazione & Anno 2010 & Anno 2014 & Var. \% \\
\hline Italia & 1.835 .605 & 1.296 .863 & $-29,35 \%$ \\
\hline USA & 1.722 .653 & 3.781 .314 & $119,51 \%$ \\
\hline Francia & 914.286 & 4.260 .720 & $366,02 \%$ \\
\hline Spagna & 839.839 & 1.564 .693 & $86,31 \%$ \\
\hline Altri & 1.215 .232 & 2.118 .806 & $74,35 \%$ \\
\hline Totale & 4.773 .490 & 13.022 .396 & $172,81 \%$ \\
\hline
\end{tabular}

\subsection{Vendite di vini rosati italiani}

Le principali regioni di produzione Italiane per il vino rosato in Norvegia sono: la Campania, il Piemonte, e l'Abruzzo. Nel periodo considerato (Tabb. 17 e 18) la crescita maggiore è registrata da Campania e Piemonte mentre il calo maggiore avviene per il veneto e l'Abruzzo.

\subsubsection{Vini rosati italiani in bag-in-box}

Il formato bag-in-box per la categoria di vino rosato sta diventando importante (Tabb. 19 e 20) raddoppiando in volume $+78,4 \%$ e fatturato $+228,4 \%$. Il formato bag-inbox rappresenta circa un terzo del volume venduto, ma il dato più significativo è nella capacità di generare fatturato che triplica in soli cinque anni.
Tabella 21. Volumi (1) vino spumante.

\begin{tabular}{|l|l|l|l|}
\hline Nazione & Anno 2010 & Anno 2014 & Var. \% \\
\hline Italia & 728.746 & 1.975 .713 & $171,11 \%$ \\
\hline Spagna & 891.198 & 987.110 & $10,76 \%$ \\
\hline Francia & 665.608 & 853.605 & $28,24 \%$ \\
\hline Australia & 155.543 & 183.472 & $17,96 \%$ \\
\hline Germania & 66.960 & 31.242 & $-53,34 \%$ \\
\hline Altri & 59.879 & 46.575 & $-22,22 \%$ \\
\hline Totale & 2.567 .934 & 4.077 .717 & $58,79 \%$ \\
\hline
\end{tabular}

Tabella 22. Fatturato (Euro) vino spumante.

\begin{tabular}{|l|l|l|l|}
\hline Nazione & Anno 2010 & Anno 2014 & Var. \% \\
\hline Italia & 13.214 .115 & 40.057 .317 & $203,14 \%$ \\
\hline Francia & 25.763 .139 & 32.499 .552 & $26,15 \%$ \\
\hline Spagna & 14.520 .539 & 18.493 .001 & $27,36 \%$ \\
\hline Australia & 2.806 .164 & 3.294 .378 & $17,40 \%$ \\
\hline Germania & 1.227 .251 & 633.101 & $-48,41 \%$ \\
\hline Altre & 1.043 .564 & 1.008 .478 & $-3,36 \%$ \\
\hline Totale & 58.574 .772 & 95.985 .827 & $63,87 \%$ \\
\hline
\end{tabular}

Tabella 23. Volume (1) vino spumante italiano.

\begin{tabular}{|l|l|l|l|}
\hline REGIONE & Volume 2010 & Volume 2014 & Var 2010-14 \\
\hline Piemonte & 351.273 & 314.682 & $-10,42 \%$ \\
\hline Veneto & 306.874 & 1.546 .033 & $403,80 \%$ \\
\hline Altre regioni & 70.599 & 113.998 & $61,47 \%$ \\
\hline Totale & 728.746 & 1.974 .713 & $170,97 \%$ \\
\hline
\end{tabular}

\section{Vini spumanti}

\subsection{Vendite complessive di vini spumanti}

La categoria dei vini spumanti nel mercato Norvegese nel periodo di riferimento (Tabb. 21 e 22) cresce in volume $+58,8 \%$ e in fatturato $+63,8 \%$ aumentando anche in valore assoluto di volume venduto.

Il trend più significativo è rappresentato dalla crescita dell'Italia in volume $+171 \%$ e in fatturato $+203 \%$. Nei volumi l'Italia passa dalla $2^{\circ}$ posizione dietro a Spagna e davanti alla Francia nel 2010 alla $1^{\circ}$ raddoppiando in volume mentre in seconda posizione si posiziona Spagna e la Francia in $3^{\circ}$.

Nel fatturato 1'Italia passa dalla $3^{\circ}$ posizione alla $1^{\circ}$, scende la Francia che si posiziona $2^{\circ}$ seguita dalla Spagna.

\subsection{Vendite di vini spumanti italiani}

Il significativo dato di crescita della categoria vino spumante per l'Italia è dato da due principali regioni di produzione; il Veneto e il Piemonte (Tabb. 23 e 24).

Specialmente il dato di crescita in volumi venduti $+403 \%$ e fatturato generati $+437 \%$ del Veneto concorrono al buon risultato Italiano nella categoria vini spumanti nel mercato norvegese, da ricercarsi soprattutto con il fenomeno del Prosecco. 
Tabella 24. Fatturato (Euro) vino spumante italiano.

\begin{tabular}{|l|l|l|l|}
\hline REGIONE & Fatturato 2010 & Fatturato 2014 & Var 2010-14 \\
\hline Veneto & 5.993 .954 & 32.199 .057 & $437,19 \%$ \\
\hline Piemonte & 5.696 .596 & 5.411 .746 & $-5,00 \%$ \\
\hline Altre regioni & 1.523 .565 & 2.446 .513 & $60,58 \%$ \\
\hline Totale & 13.214 .115 & 40.057 .316 & $203,14 \%$ \\
\hline
\end{tabular}

\section{Conclusioni}

Nel quinquennio 2010-2014 l'Italia è il paese che cresce maggiormente nel mercato del vino Norvegese.

L'incremento si registra per tutte le categorie di vino sia in termini di fatturato che di volume, permettendo così all'Italia di posizionarsi tra i principali paesi esportatori in ogni categoria.

Nella categoria vino rosso l'aumento del $23 \%$ in volume e del $35 \%$ in fatturato evidenzia la capacità dei vini italiani di andare in controtendenza rispetto i principali competitor, grazie anche alle ottime performance del formato bag-in-box.

Anche nella categoria vini bianchi l'Italia incrementa le quote di mercato crescendo in volume del $22 \%$ ed in fatturato del $39 \%$.
Il vino italiano rosato inizia a farsi apprezzare e i margini di crescita rimangono incoraggianti verso i naturali competitor di questa categoria in cui comunque cresce del $16,3 \%$ in volume e del $4 \%$ in fatturato.

Nel vino spumante la crescita è del tutto evidente: volumi e fatturato sono più che raddoppiati, andando così a superare storici competitor come la Francia; soprattutto grazie al caso "Prosecco" che anche nel mercato Norvegese supera denominazioni storiche come "Champagne" e "Cava".

Il mercato Norvegese si conferma quindi molto dinamico $\mathrm{e}$ in continua crescita nei consumi e diventerà sempre più strategico per il mercato vinicolo Italiano che dovrà difendere nei prossimi anni le quote di mercato conquistate nell'ultimo quinquennio.

\section{Bibliografia}

[1] M. B. Lai, A. Cavicchi, K Rickertsen., A. M., Corsi, L. Casini, (2013). Monopoly and wine: the Norwegian case. British Food Journal, 115(2), 314326

[2] M. B. Lai, I. Etzo, and C. Massidda. "ANGERS 2011" 